\title{
A Comparison of Three PML Treatments for CAA (and CFD)
}

\author{
John W. Goodrich * \\ Glenn Research Center, Cleveland, Ohio, 44135, USA
}

\begin{abstract}
In this paper we compare three Perfectly Matched Layer (PML) treatments by means of a series of numerical experiments, using common numerical algorithms, computational grids, and code implementations. These comparisons are with the Linearized Euler Equations, for base uniform base flow. We see that there are two very good PML candidates, and that can both control the introduced error. Furthermore, we also show that corners can be handled with essentially no increase in the introduced error, and that with a good PML, the outer boundary is the most significant source of error.
\end{abstract}

\section{Introduction}

Aeroacoustics research is conducted with the combined use of theoretical analysis, experimental observations, and computation. Computational AeroAcoustics (CAA) is growing as a result of increases in the capability of numerical methods and computer hardware, and is increasingly valuable both as a source of insight and as a tool for design [3, 17, 22, 27]. Artificial boundary treatments are a critical element for CAA because they permit the restriction of a numerical domain while causing an error in the numerical solution that is intended to be limited. Various reviews of the extensive work done to date on this problem have been published, including $[2,5,6,12,14,15,23,26]$.

Hagstrom $[13,18,4]$ has analytically developed a PML treatment for artificial radiation boundaries in the case of the LEE with uniform base flow. A widespread and general alternative approach is to use a sponge zone, as in [1, 19, 20,21]. Karni [16] discusses possible forms for damping in a reflectionless sponge zone for a first order hyperbolic system. In this paper we use two PML sponge layer treatments. The two sponge layer treatments can be extended to the LEE with nonuniform steady and unsteady base flows, and to the Compressible Navier Stokes Equations. These three PML treatments are compared by means of a series of numerical experiments, using common numerical algorithms, computational grids, and code implementations. Two further series of experiments with one of the sponge layer treatments show how to treat corners where two PMLs overlap, and that the outer boundary treatment can cause large errors even with a good PML treatment. The numerical experiments are designed to isolate three aspects that are important for the actual use of the PML: (1) a simple PML at a single boundary, with a layer that is wide enough so that the propagated disturbance does not reach the outer boundary; (2) a combination of two PMLs at a common corner, with a layer that is wide enough so that the propagated disturbance does not reach the outer boundary; and (3) a PML combined with a treatment for the outer boundary of the layer. Within the context of these examples, we see that there are two very good PML candidates that can both control the introduced error, that corners can be handled with essentially no increase in the introduced error, and that with a good PML, the outer boundary is the most significant source of error.

*Acoustics Branch, MS 54-3, or John.W.Goodrich@nasa.gov. 


\section{PML for the LEE in two space dimensions}

The Linearized Euler Equations (LEE) are obtained by linearizing the Euler Equations about a base solution. We will consider two dimensional cases where the base solution $\left\{p_{b}, u_{b}, v_{b}, \rho_{b}\right\}$ is steady. We use a nondimensional form of the governing equations, and note that a steady parallel flow with constant pressure and density is a solution for the Euler Equations.

The simplest case for the LEE in 2D is with a constant base solution. We express the constant base velocity components are expressed in terms of Mach number, $\left(u_{b}, v_{b}\right)=\left(M_{x}, M_{y}\right)$. The perturbation density is uncoupled from the perturbation pressure and velocity, and will be neglected. In this case, the nondimensional form of the LEE for perturbation pressure and velocity components can be written as

$$
\begin{gathered}
\frac{\partial p}{\partial t}+M_{x} \frac{\partial p}{\partial x}+M_{y} \frac{\partial p}{\partial y}+\frac{\partial u}{\partial x}+\frac{\partial v}{\partial y}=0 \\
\frac{\partial u}{\partial t}+M_{x} \frac{\partial u}{\partial x}+M_{y} \frac{\partial u}{\partial y}+\frac{\partial p}{\partial x}=0 \\
\frac{\partial v}{\partial t}+M_{x} \frac{\partial v}{\partial x}+M_{y} \frac{\partial v}{\partial y}+\frac{\partial p}{\partial y}=0 .
\end{gathered}
$$

If we let the unsteady perturbation quantities be

$$
\vec{U}=(p, u, v)^{T},
$$

then this simplest form of the LEE in two space dimensions can be written as

$$
\frac{\partial \vec{U}}{\partial t}+A \frac{\partial \vec{U}}{\partial x}+B \frac{\partial \vec{U}}{\partial y}=0
$$

where $A$ and $B$ are the constant matrices

$$
A=\left(\begin{array}{ccc}
M_{x} & 1 & 0 \\
1 & M_{x} & 0 \\
0 & 0 & M_{x}
\end{array}\right), \text { and } B=\left(\begin{array}{ccc}
M_{y} & 0 & 1 \\
0 & M_{y} & 0 \\
1 & 0 & M_{y}
\end{array}\right)
$$

A Perfectly Matched Layer (PML) boundary treatment is designed for this system by ensuring that the plane wave solutions for the LEE in the numerical domain perfectly match the plane wave solutions for the adjacent PML domain along the interface between them.

We shall consider the LEE with a pressure source term, with the modified pressure equation

$$
\frac{\partial p}{\partial t}+M_{x} \frac{\partial p}{\partial x}+M_{y} \frac{\partial p}{\partial y}+\frac{\partial u}{\partial x}+\frac{\partial v}{\partial y}=\alpha \sin [2 \pi \nu t] \exp \left[-\left(\beta_{x} x^{2}+\beta_{y} y^{2}\right)\right]=g(x, y, t),
$$

for all $(x, y)$ in the numerical domain. With this source term, the LEE can be written as

$$
\frac{\partial \vec{U}}{\partial t}+A \frac{\partial \vec{U}}{\partial x}+B \frac{\partial \vec{U}}{\partial y}=\vec{G}
$$

where $\vec{G}=(g, 0,0)^{T}$ inside the numerical domain. For the numerical experiments reported in this paper, we always take

$$
\alpha=0.01, \quad \beta_{x}=36=\beta_{y}, \quad \nu=1 .
$$

Note that $\exp \left[-36 * 2^{2}\right] \approx 10^{-63}$. This radiating source produces a propagating disturbance that is $O\left[10^{-4}\right]$ in the numerical domains that we use. We do not include the source term in the pressure equation inside the PML, and take a large enough numerical domain so that the pressure source and all of its relevant derivatives are effectively 0 at the interface. 


\subsection{The Hagstrom PML}

Hagstrom [13] has formulated a PML boundary treatment for this system, which has been further investigated by Motamed [18] and Fröling [4]. For an artificial boundary layer that is perpendicular to the $x$ axis, this PML treatment can be expressed as the system

$$
\begin{gathered}
\frac{\partial \vec{U}}{\partial t}+A\left(\frac{\partial \vec{U}}{\partial x}+\mu \sigma(x) \vec{U}\right)+B \frac{\partial \vec{U}}{\partial y}+\vec{W}=0 \\
\frac{\partial \vec{W}}{\partial t}+M_{y} \frac{\partial \vec{W}}{\partial y}+\sigma(x) \vec{W}+\sigma(x) A\left(\frac{\partial \vec{U}}{\partial x}+\mu \sigma(x) \vec{U}\right)=0,
\end{gathered}
$$

where $\vec{W}=\left(w_{p}, w_{u}, w_{v}\right)^{T}$ is a vector of auxiliary variables, $\sigma(x)$ is a damping profile, and

$$
\mu=\frac{M_{x}}{1-M_{x}^{2}} .
$$

This PML system is used in a layer that is joined or matched to the computational domain of interest. If the numerical domain is $(x, y) \in\left[X_{l}, X_{r}\right] \times\left[Y_{b}, Y_{t}\right]$, then a PML domain on the right hand boundary would be for $(x, y) \in\left[X_{r}, X_{r}+D_{r}\right] \times\left[Y_{b}, Y_{t}\right]$, where $D_{r}$ is the layer width. In such a layer, a typical damping profile from $[4]$ is

$$
\sigma(x)=\alpha\left(\frac{x-X_{r}}{D_{r}}\right)^{n},
$$

where $\alpha$ is a constant, and usually $n=3,4$, or 5 . We shall use

$$
\sigma(x)=c \frac{\left(x-X_{r}\right)^{6}}{\left(1+\left(x-X_{r}\right)^{2}\right)^{3}},
$$

and will call the Hagstrom PML with this damping profile PML A.

\subsection{The Karni directional damping PML}

Karni [16] has given a very nice eigensystem analysis of PML treatments with filtering operators for both slowing down and damping outgoing waves. By considering diagonalization in a general direction, Karni gives one particular form for directional damping in an artificial boundary layer as the system

$$
\frac{\partial \vec{U}}{\partial t}+A \frac{\partial \vec{U}}{\partial x}+B \frac{\partial \vec{U}}{\partial y}+\delta(\cos (\theta) A+\sin (\theta) B) \vec{U}=0
$$

where $\theta$ is the angle of the damping direction, and $\delta$ is the damping rate. For a damping PML perpendicular to the $x$ axis, this reduces to the system

$$
\frac{\partial \vec{U}}{\partial t}+A \frac{\partial \vec{U}}{\partial x}+B \frac{\partial \vec{U}}{\partial y} \pm \delta A \vec{U}=0
$$

where $+\delta$ is taken on the right, and $-\delta$ is taken on the left. This PML system with $\delta=\sigma$ will be called PML B. We note that the damping terms are

$$
d_{p}= \pm \sigma\left(M_{x} p+u\right), \quad d_{u}= \pm \sigma\left(M_{x} u+p\right), \text { and } d_{v}= \pm \sigma M_{x} v
$$

in the equations for $p, u$, and $v$, respectively, where + is taken on the right, and - on the left. Notice that if $M_{x}=0$, then the damping terms for $p$ and $u$ are proportional to each other, and that $v$ is undamped. 


\subsection{A simple PML by analogy}

The simplest example of a decaying dynamic is radioactive decay, governed by the ordinary differential equation

$$
\frac{d f}{d t}+c f=0
$$

with $c>0$. If we consider the LEE as an evolutionary operator that is analogous to a time derivative, then by analogy a simple damping system is

$$
\frac{\partial \vec{U}}{\partial t}+A \frac{\partial \vec{U}}{\partial x}+B \frac{\partial \vec{U}}{\partial y}+\sigma \vec{U}=0
$$

We shall call this PML treatment PML C. We note that the damping terms are

$$
d_{p}=\sigma p, \quad d_{u}=\sigma u, \text { and } d_{v}=\sigma v,
$$

in the equations for $p, u$, and $v$, respectively. Notice that these damping terms are independent from the underlying steady flow, and that the $p$ and $u$ terms are the opposite of the damping terms in PML B for the case $M_{x}=0$. If

$$
\sigma_{R}=\frac{\sigma}{M_{x}+1}, \quad \sigma_{L}=\frac{\sigma}{M_{x}-1}, \text { and } \sigma_{v}=\frac{\sigma}{M_{x}},
$$

then PML C is obtained from the characteristic analysis of a general damping form. What we call PML C has been discussed in [20], where the damping term $\sigma \vec{U}$ is called a relaxation source term, and the damping coefficient $\sigma=1 / \tau$, where $\tau$ is a relaxation time.

\subsection{Characteristic based PML}

Karni [16] observes that the damping rate need not be the same for the different eigenfunctions of the hyperbolic system, but that the damping terms should not alter the eigenvectors of the hyperbolic system with respect to the damping direction. A characteristic analysis similar to Thompson's $[24,25]$ can be used to obtain a general damping layer. The characteristic variables or Riemann invariants for the LEE diagonalized in the $x$ direction are

$$
R=p+u, \quad L=p-u, \text { and } v,
$$

with propagation speeds $M_{x}+1, M_{x}-1$, and $M_{x}$, respectively. If $0 \leq M_{x}<1$, then $R$ is right going, $L$ is left going, and $v$ is not left going. The LEE with damping terms can be expressed in terms of these variables as

$$
\begin{gathered}
\frac{\partial R}{\partial t}+\left(M_{x}+1\right) \frac{\partial R}{\partial x}+M_{y} \frac{\partial R}{\partial y}+\frac{\partial v}{\partial y}+\sigma_{R}\left(M_{x}+1\right) R=0, \\
\frac{\partial L}{\partial t}+\left(M_{x}-1\right) \frac{\partial L}{\partial x}+M_{y} \frac{\partial L}{\partial y}+\frac{\partial v}{\partial y}+\sigma_{L}\left(M_{x}-1\right) L=0, \\
\frac{\partial v}{\partial t}+M_{x} \frac{\partial v}{\partial x}+M_{y} \frac{\partial v}{\partial y}+\frac{1}{2} \frac{\partial R}{\partial y}+\frac{1}{2} \frac{\partial L}{\partial y}+\sigma_{v} M_{x} v=0,
\end{gathered}
$$

where $\sigma_{R}, \sigma_{L}$, and $\sigma_{v}$ need not be the same. The damping terms for $R, L$, and $v$ are

$$
d_{R}=\sigma_{R}\left(M_{x}+1\right) R, \quad d_{L}=\sigma_{L}\left(M_{x}-1\right) L, \text { and } d_{v}=\sigma_{v} M_{x} v
$$

respectively. If this form of the equations is recast in terms of the primitive variables, then the damping terms in the primitive variable equations are

$$
\begin{aligned}
& d_{p}=\left(\frac{\sigma_{R}+\sigma_{L}}{2} M_{x}+\frac{\sigma_{R}-\sigma_{L}}{2}\right) p+\left(\frac{\sigma_{R}-\sigma_{L}}{2} M_{x}+\frac{\sigma_{R}+\sigma_{L}}{2}\right) u=a p+b u, \\
& d_{u}=\left(\frac{\sigma_{R}+\sigma_{L}}{2} M_{x}+\frac{\sigma_{R}-\sigma_{L}}{2}\right) u+\left(\frac{\sigma_{R}-\sigma_{L}}{2} M_{x}+\frac{\sigma_{R}+\sigma_{L}}{2}\right) p=a u+b p,
\end{aligned}
$$


and

$$
d_{v}=\sigma_{v} M_{x} v=c v,
$$

respectively. The LEE with these primative variable damping terms can be written as

$$
\frac{\partial \vec{U}}{\partial t}+A \frac{\partial \vec{U}}{\partial x}+B \frac{\partial \vec{U}}{\partial y}+D \vec{U}=0
$$

with the symmetric damping matrix $D$ is

$$
D=\left(\begin{array}{ccc}
\left(\frac{\sigma_{R}+\sigma_{L}}{2} M_{x}+\frac{\sigma_{R}-\sigma_{L}}{2}\right) & \left(\frac{\sigma_{R}-\sigma_{L}}{2} M_{x}+\frac{\sigma_{R}+\sigma_{L}}{2}\right) & 0 \\
\left.\frac{\sigma_{R}-\sigma_{L}}{2} M_{x}+\frac{\sigma_{R}+\sigma_{L}}{2}\right) & \left(\frac{\sigma_{R}+\sigma_{L}}{2} M_{x}+\frac{\sigma_{R}-\sigma_{L}}{2}\right) & 0 \\
0 & 0 & \sigma_{v} M_{x}
\end{array}\right)=\left(\begin{array}{ccc}
a & b & 0 \\
b & a & 0 \\
0 & 0 & c
\end{array}\right) .
$$

If we consider the primitive damping coefficients $a, b$, and $c$ to be independant, then

$$
a+b=\sigma_{R}\left(M_{x}+1\right), \quad a-b=\sigma_{L}\left(M_{x}-1\right), \text { and } c=\sigma_{v} M_{x} .
$$

The eigenvectors for $A$ are: $E_{R}=(1,1,0)^{T}$ with eigenvalue $\lambda_{A R}=M_{x}+1 ; E_{L}=(1,-1,0)^{T}$ with eigenvalue $\lambda_{A L}=M_{x}-1$; and $E_{v}=(0,0,1)^{T}$ with eigenvalue $\lambda_{A v}=M_{x}$. The eigenvectors for $D$ are the same as for $A$, with eigenvalues

$$
\begin{aligned}
& \lambda_{D R}=a+b=\sigma_{R}\left(M_{x}+1\right)=\sigma_{R} \lambda_{A R}, \\
& \lambda_{D L}=a-b=\sigma_{L}\left(M_{x}-1\right)=\sigma_{L} \lambda_{A L},
\end{aligned}
$$

and

$$
\lambda_{D v}=c=\sigma_{v} M_{x}=\sigma_{v} \lambda_{A v},
$$

respectively.

Both PML B and PML C use just one damping profile, with $\sigma_{R}=\sigma_{L}=\sigma_{v}=\sigma$. If we set $a=M_{x} \sigma=c$, and $b=\sigma$, then PML B is recovered, with

$$
D_{B}=\sigma(x)\left(\begin{array}{ccc}
M_{x} & 1 & 0 \\
1 & M_{x} & 0 \\
0 & 0 & M_{x}
\end{array}\right)
$$

If $\sigma \geq 0$ in a PML B, then $R$ is damped as it goes right, $L$ is amplified as it goes left, and $v$ is either damped if $M_{x}>0$, or unchanged if $M_{x}=0$. Note that if $M_{x}=0$, then the damping matrix for PML B becomes

$$
\left.D_{B}\right|_{M_{x}=0}=\sigma(x)\left(\begin{array}{ccc}
0 & 1 & 0 \\
1 & 0 & 0 \\
0 & 0 & 0
\end{array}\right)
$$

If $a=\sigma=c$ and $b=0$, then PML C is recovered, with

$$
D_{C}=\sigma(x)\left(\begin{array}{lll}
1 & 0 & 0 \\
0 & 1 & 0 \\
0 & 0 & 1
\end{array}\right)
$$

If $\sigma \geq 0$ in a PML C, then $R, L$ and $v$ are all damped at the same rate. For a PML perpendicular to the $y$ coordinate axis, we note that

$$
E=\left(\begin{array}{lll}
d & 0 & e \\
0 & f & 0 \\
e & 0 & d
\end{array}\right)
$$

is an acceptible general form for a damping matrix similar to $D$ for the case in the $x$ direction. 


\section{Numerical Algorithm}

The Hermite/Cauchy-Kovalevsky/Taylor method (see [7], [8], [9], [10], [11]) is used for all of the experiments that are reported in this paper. This method uses two staggered uniform grids, offset by half a grid spacing in both $x$ and $y$. The first or base grid is used for initial data, and the numerical solution at each full time step. The second or offset grid is used for the numerical solution at each half time step. The time evolution through a half time step from one grid to the next starts with multidimensional Hermite spatial interpolation of the local data on the current grid stencil, with a tensor interpolant for a rectangular grid, it then uses the governing equations for Cauchy-Kovalevsky recursion to produce all of the required time derivatives in space and time from the spatial derivatives produced by the interpolant, and it ends by propagating through a half time step with a Taylor series. This approach is not simply an algorithm, but is actually a method for developing or specifying numerical algorithms. This approach has had various names, but in this paper, we will call it the Hermite/Cauchy-Kovalevsky/Taylor method, or the HCKT method.

The spatial interpolant with data on one grid is expanded about the center of the stencil, which is a point in the alternate grid, so that the spatial derivatives from the interpolant with known data on one grid are used by the Cauchy-Kovalevsky recursion to compute time derivatives at a grid point of the alternate grid. A simple method is used for the numerical experiments that are reported in this paper. This method uses a $2 \times 2$ local stencil, and at each of the four grid points in the stencil, for each of the dependant variables, we carry data for the variable itself, and its $x, y$, and $x y$ derivatives. The spatial interpolant is a third order Hermite tensor interpolant on a stencil that is two points wide, and it simultaneously and consistently estimates all the derivatives of order $\{(m, n): 0 \leq m, n \leq 3\}$, with up to the sixth order $(3,3)$ cross derivative. Other methods could be specified with different stencils and different data, but we use only one simple method since the PML issues are the focus of interest here.

The Cauchy-Kovalevsky recursion is used with the local interpolated data surface considered to be initial data, and it can produce time derivative data of any order. The recursion formula comes directly from the governing equations, and in the simple constant coefficient case, it is

$$
\frac{\partial^{i+j+k+1} \vec{U}}{\partial x^{i} \partial y^{j} \partial t^{k+1}}=-A \frac{\partial^{i+j+k+1} \vec{U}}{\partial x^{i+1} \partial y^{j} \partial t^{k}}-B \frac{\partial^{i+j+k+1} \vec{U}}{\partial x^{i} \partial y^{j+1} \partial t^{k}}
$$

for $k \geq 0$, and for all required $i$ and $j$, depending on the interpolant and the upper range of $k$. The time derivatives that are obtained from this recursion are used in a simple Taylor series time advancement at the stencil center, so that known data on one grid produces time derivaties for a solution on the alternate grid. For the LEE with constant coefficients, and with the method that we use that has a third order interpolant, if the recursion is carried through the sixth order in time, and if all of the time derivatives are used for the Taylor series time advancement, then the locally interpolated two dimensional data surface would be propagated in time exactly. Since the propagation of the local two dimensional data surface is exact, this method correctly captures propagation along characteristic surfaces in multiple spatial dimensions, and in this sense, it can be said to be a correct generalization of the method of characteristics to higher dimensions. For the results presented here, we only compute and use time derivatives through the fourth order for the third order interpolant, so that the propagation is not exact, but it still is correctly along the characteristic surfaces to the fourth order in time. The numerical method is third order in time and space for $p$, $u$, and $v$, it is second order for their $x$ and $y$ derivatives, and it is first order for their $x y$ cross derivative. Overall, this method is third order in time and space for the physical variables, with diffusive leading order error terms.

By changing the stencil width, or the depth of data at each stencil point, various other interpolants can be used, with arbitrary order. By changing the recursion to a different set of governing equations, a different physical system can be simulated. Any system of partial differential equations can be approximated with this HCKT algorithm development method as long as the governing equations can be written in the form

$$
\frac{\partial \vec{V}}{\partial t}=F(\vec{V})
$$

where $V$ is the vector of dependant variables, and where $F$ is any partial differential operator defined in terms of $\vec{V}$ and its spatial partial derivatives. For example, the various PML equation systems specify governing equations in the layers that are different from the LEE that govern in the numerical domain. 
We have used the HCKT method to develop algorithms for a variety of governing equations, including the advection equation, the heat equation, the convection diffusion equation, a first order dispersive system, the LEE, Burger's equation, and the Compressible Navier-Stokes Equatiob, and these algorithms have had accuracy up to the twenty first order in both space and time. Algorithms can be specified by the HCKT method by simply replacing the recursion equations or subroutine. A comparison of accuracy and efficiency for algorithms up to the fifteenth order for a simple one dimensional problem is given in [10].

\section{Experiments With One PML Boundary}

In this section we presents results from a series of numerical experiments using PML A, B, and C. The computations that we present here are all on a rectangular numerical domain,

$$
\Omega_{N}=\left\{(x, y) \in\left[X_{l}, X_{r}\right] \times\left[Y_{b}, Y_{t}\right]\right\},
$$

with an additional PML layer on the right

$$
\Omega_{R}=\left\{(x, y) \in\left[X_{r}, X_{r}+D_{r}\right] \times\left[Y_{b}, Y_{t}\right]\right\},
$$

where $D_{r}$ is the layer width. All of these computations are for the LEE with a Gaussian pressure source. The governing equations in $\Omega_{N}$ are

$$
\frac{\partial \vec{U}}{\partial t}+A \frac{\partial \vec{U}}{\partial x}+B \frac{\partial \vec{U}}{\partial y}=\vec{G}=(g, 0,0)^{T},
$$

where

$$
g=0.01 \sin (2 \pi t) \exp \left(-36\left(x^{2}+y^{2}\right)\right) .
$$

The source is negligible except near the origin, it radiates a signal away from the origin, and it is not included in the PML equations. Since we have an interest in acoustic applications, we consider the propagation medium to be scaled by the standard atmosphere, with the pressure of the base flow to be one atmosphere, and the amplitude of the pressure disturbance has been chosen to produce a radiating signal that is $O\left[10^{-4}\right]$. The frequency is $\nu=1$, so that the wavelength of the radiated sound is one spatial unit. If we consider the unit time to represent $1 \mathrm{~s}$ and the frequency to represent $1 \mathrm{~Hz}$, then the spatial unit corresponds to approximately $340 \mathrm{~m}$, while if we consider the spatial unit to correspond to $1 \mathrm{~m}$, then the time unit scales so that $\nu=1$ corresponds to a frequency of approximately $340 \mathrm{~Hz}$. The numerical domain is always chosen large enough, and the elapsed simulation time short enough, so that in these particular experiments, the radiated signal only leaves the numerical domain on the right at the interface between the numerical domain $\Omega_{N}$ and the PML layer $\Omega_{R}$. In other words, we use Big Enough Domain (BED) boundary conditions along the numerical domain limits at $X_{l}, Y_{b}$ and $Y_{t}$. The damping profile that we use is

$$
\sigma(x)=c \frac{\left(x-x_{R}\right)^{6}}{\left(1+\left(x-x_{R}\right)^{2}\right)^{3}},
$$

for $X_{r} \leq x \leq X_{r}+D_{r}$, where $D_{r}$ is chosen large enough so that we have a BED boundary condition at $X_{r}+D_{r}$, and no signal exits the PML layer $\Omega_{R}$ at its outer boundary. Note that we are now using $c$ as a real constant, and not as a function in a damping matrix, as earlier. A third order algorithm is used, and the damping profile has been chosen first of all so that $\sigma$ and sufficient of its derivatives are 0 at the interface between the numerical domain and the PML layer to ensure a completely smooth seam along the interface, through the order of the leading order truncation error terms of the numerical algorithm. This means that one sided characteristic matching at the interface is unneccesary, since there is no difference up to the order of the numerical method between the LEE and the PML system at the interface. We simply change the recursion routine from $\Omega_{N}$ to $\Omega_{R}$, and we use the numerical domain recursion on the interface at the half time steps when a cell center is at $x_{R}$. The damping profile has also been chosen to smoothly vary from 0 along the interface to an essentially constant value deeper inside the PML layer. All computations are with

$$
\triangle x=\frac{1}{24}=\triangle y, \quad \triangle t=\frac{1}{48} .
$$


In all cases, the reported data has been obtained by comparison with a reference solution computed with $c=0$, so that the PML layer $\Omega_{R}$ is actually just an extension of the numerical domain $\Omega_{N}$, with a BED boundary condition at the outer boundary of the layer. We have always taken $X_{r}=2$, with data obtained as

$$
E_{L}(t)=\max \left\{\left|p_{L}(x, y, t)-p_{\text {ref }}(x, y, t)\right|:(x, y) \in \Omega_{N}\right\}
$$

where $p_{\text {ref }}$ is the pressure from the reference solution, and $p_{L}$ is the solution using PML $L$, for $L=\mathrm{A}$, $B$, or $C$. The reported data is not an error in the sense of a comparison of a numerical estimate and an exact solution, but it is a measure of the difference betweeen two numerical estimates, one on a Big Enough Domain, and one with a PML. The reported data indicates only the effect on a numerical solution that is produced by truncating the numerical domain with a particular PML.

Table 1: Maximum absolute disturbance from one PML on the right.

PML A, B, and C with $\triangle x=1 / 24=\triangle y, \triangle t=1 / 48$. Data with $M_{x}=0.0,0.4$, or 0.8 at $t=10$.

\begin{tabular}{|r|lll|}
\hline $\mathrm{c}$ & $E_{A}(10)$ & $E_{B}(10)$ & $E_{C}(10)$ \\
\hline \hline & & $M_{x}=0.0$ & \\
\hline 0.001 & $5.7686 \times 10^{-14}$ & $7.3605 \times 10^{-12}$ & $6.4692 \times 10^{-9}$ \\
\hline 0.010 & $5.7710 \times 10^{-13}$ & $7.3643 \times 10^{-11}$ & $6.4552 \times 10^{-8}$ \\
\hline 0.100 & $5.7946 \times 10^{-12}$ & $7.4020 \times 10^{-10}$ & $6.3175 \times 10^{-7}$ \\
\hline 1.000 & $6.0052 \times 10^{-11}$ & $7.7960 \times 10^{-9}$ & $5.2127 \times 10^{-6}$ \\
\hline 10.00 & $6.7827 \times 10^{-10}$ & $1.5901 \times 10^{-7}$ & $2.7457 \times 10^{-5}$ \\
\hline \hline & \multicolumn{3}{|c|}{$M_{x}=0.4$} \\
\hline 0.001 & $7.1998 \times 10^{-13}$ & $1.4915 \times 10^{-12}$ & $2.9545 \times 10^{-9}$ \\
\hline 0.010 & $7.2031 \times 10^{-12}$ & $1.4934 \times 10^{-11}$ & $2.9506 \times 10^{-8}$ \\
\hline 0.100 & $7.2357 \times 10^{-11}$ & $1.5122 \times 10^{-10}$ & $2.9115 \times 10^{-7}$ \\
\hline 1.000 & $7.5906 \times 10^{-10}$ & $1.7337 \times 10^{-9}$ & $2.5720 \times 10^{-6}$ \\
\hline 10.00 & $1.0285 \times 10^{-8}$ & $7.9125 \times 10^{-8}$ & $1.4206 \times 10^{-5}$ \\
\hline \hline & \multicolumn{3}{|}{$M_{x}=0.8$} \\
\hline 0.001 & $1.3805 \times 10^{-13}$ & $6.0922 \times 10^{-14}$ & $3.3349 \times 10^{-10}$ \\
\hline 0.010 & $1.3848 \times 10^{-12}$ & $6.1014 \times 10^{-13}$ & $3.3219 \times 10^{-9}$ \\
\hline 0.100 & $1.4283 \times 10^{-11}$ & $6.1966 \times 10^{-12}$ & $3.2596 \times 10^{-8}$ \\
\hline 1.000 & $1.8633 \times 10^{-10}$ & $7.1884 \times 10^{-11}$ & $2.9821 \times 10^{-7}$ \\
\hline 10.00 & Blew Up & $3.0027 \times 10^{-9}$ & $1.9087 \times 10^{-6}$ \\
\hline \hline
\end{tabular}

The computations reported in Table 1 are for three cases of uniform mean flow, with $M_{x}=0.0,0.4$, or 0.8. In the first case with $M_{x}=0$, the evolution of $p$ is also governed by the standard wave equation. In all cases the reported data is at $t=10$. The numerical domains and PML layers are

$$
\begin{aligned}
& \Omega_{N}=[-11,2] \times[-11,11], \text { and } \Omega_{R}=[2,11] \times[-11,11], \text { for } M_{x}=0.0, \\
& \Omega_{N}=[-7,3] \times[-11,11], \text { and } \Omega_{R}=[3,15] \times[-11,11], \text { for } M_{x}=0.4, \\
& \Omega_{N}=[-3,4] \times[-11,11], \text { and } \Omega_{R}=[4,19] \times[-11,11], \text { for } M_{x}=0.8 .
\end{aligned}
$$

In order to scale the data in Table 1, the pressure solutions with the expanded BED numerical domains are $O\left[10^{-4}\right]$ in all cases. Notice that the disturbance of the numerical solution that is produced by introducing a PML ranges in absolute terms from $O\left[10^{-14}\right]$ to $O\left[10^{-6}\right]$, or in relative terms from $O\left[10^{-10}\right]$ to $O\left[10^{-2}\right]$. None of the disturbances in the numerical domain solution can be seen at the scale of the solution if they are relatively smaller than $O\left[10^{-3}\right]$. We note the evident superiority of PML A and B when compared to the simple PML C. We also note that PML A works better at lower convection speeds, while PML B works better at higher convection speeds. From this series of comparisons it appears on the whole that PML A and PML B are reasonably comparable, with the advantage of PML A at lower speeds being somewhat 
better than the advantage of PML B at higher speeds. Recall that PML A uses a set of auxiliary variables and equations in the PML, while PML B uses only the primitive variables, so that PML A requires twice the amount of storage and has twice as many equations in the PML layer. Also recall that PML A is specifically taylored for the LEE with constant coefficients, or for uniform flow, while PML B can be readily extended to nonuniform or even unsteady flows by using variable coefficients in the PML B system. The numerical domain solution disturbances scale with the damping coefficient $c$, and the effect of the PML can be made essentially as small as desired with a small enough damping coefficient. This shows that these PML treatments actually do smoothly match across the interface, and that their effect on the solution in the numerical domain is controllable. We must recall that using a smaller damping coefficient to control the numerical domain disturbance will require a wider layer, so that getting a more accurate solution has a cost, as is only to be expected.

\section{Treating a Corner Between Two PML Boundaries}

Treating a corner between two PML boundaries has been a distinct problem with its own difficulties. We note again that Karni [16] has given a particular form for directional damping in an artificial boundary layer as the system

$$
\frac{\partial \vec{U}}{\partial t}+A \frac{\partial \vec{U}}{\partial x}+B \frac{\partial \vec{U}}{\partial y}+\delta(\cos (\theta) A+\sin (\theta) B) \vec{U}=0
$$

where $\theta$ is the direction of damping, and $\delta$ is the damping rate. Notice here that $\delta$ is the same damping rate for both $A \vec{U}$ and $B \vec{U}$. For damping in the direction of a coordinate axis, this directional form reduces to PML B in that direction. We consider the corner between a PML B in $x$ on the right, with domain $\Omega_{R}=\left[X_{R}, X_{R}+D_{R}\right] \times\left[Y_{B}, Y_{T}\right]$ and equations

$$
\frac{\partial \vec{U}}{\partial t}+A \frac{\partial \vec{U}}{\partial x}+B \frac{\partial \vec{U}}{\partial y}+\sigma_{R}(x) A \vec{U}=0
$$

and a PML B in $y$ on the top, with domain $\Omega_{T}=\left[X_{L}, X_{R}\right] \times\left[Y_{T}, Y_{T}+D_{T}\right]$ and equations

$$
\frac{\partial \vec{U}}{\partial t}+A \frac{\partial \vec{U}}{\partial x}+B \frac{\partial \vec{U}}{\partial y}+\sigma_{T}(y) B \vec{U}=0
$$

Note that $\sigma_{R}(x)$ and $\sigma_{T}(y)$ need not have the same functional form. In this upper right corner we use a blended PML B form, with domain $\Omega_{R T}=\left[X_{R}, X_{R}+D_{R}\right] \times\left[Y_{T}, Y_{T}+D_{T}\right]$ and equations

$$
\frac{\partial \vec{U}}{\partial t}+A \frac{\partial \vec{U}}{\partial x}+B \frac{\partial \vec{U}}{\partial y}+\frac{\left(x-x_{R}\right) \sigma_{R}(x)}{\sqrt{\left(x-x_{R}\right)^{2}+\left(y-y_{T}\right)^{2}}} A \vec{U}+\frac{\left(y-y_{T}\right) \sigma_{T}(y)}{\sqrt{\left(x-x_{R}\right)^{2}+\left(y-y_{T}\right)^{2}}} B \vec{U}=0 .
$$

This blended PML B form in $\Omega_{R T}$ is not a directional damping except along the bottom and left edges of the corner PML domain, even though

$$
\cos (\theta(x, y))=\frac{\left(x-x_{R}\right)}{\sqrt{\left(x-x_{R}\right)^{2}+\left(y-y_{T}\right)^{2}}}, \quad \sin (\theta(x, y))=\frac{\left(y-y_{T}\right)}{\sqrt{\left(x-x_{R}\right)^{2}+\left(y-y_{T}\right)^{2}}}
$$

where $\theta(x, y)$ is the angle to the point $(x, y)$ relative to the $\Omega_{R T}$ corner point $\left(X_{R}, Y_{T}\right)$. Many PML treatments can be succsefully blended like this at a corner, for example, any of the PML with the general characteristic form dicussed above, and in particular PML C. It is not immediately clear how to implement a blended corner treatment like this for PML A with its auxiliary functions.

The computations reported in Table 2 are all for a uniform mean flow, with columns for each of the cases $\left(M_{x}, M_{y}\right)=(0.0,0.0),(0.4,0.0),(0.8,0.0)$, and $(0.8,0.4)$. In the last case, the convection speed is $\left|\left(M_{x}, M_{y}\right)\right| \approx 0.89$, and is still subsonic. The numerical domain is

$$
\Omega_{N}=\left[X_{l}, X_{r}\right] \times\left[Y_{b}, Y_{t}\right]
$$


Table 2: Maximum absolute disturbance at $t=10$ from a top right blended corner.

PML B on the right and the top with $\triangle x=1 / 24=\triangle y, \triangle t=1 / 48$.

\begin{tabular}{|r|llll|}
\hline $\mathrm{c}$ & $\left(M_{x}, M_{y}\right)=(0,0)$ & $\left(M_{x}, M_{y}\right)=(.4,0)$ & $\left(M_{x}, M_{y}\right)=(.8,0)$ & $\left(M_{x}, M_{y}\right)=(.8, .4)$ \\
\hline \hline 0.001 & $7.3605 \times 10^{-12}$ & $6.5968 \times 10^{-12}$ & $4.7396^{-12}$ & $9.6336^{-13}$ \\
\hline 0.010 & $7.3643 \times 10^{-11}$ & $6.5988 \times 10^{-11}$ & $4.7413^{-11}$ & $9.6505^{-12}$ \\
\hline 0.100 & $7.4020 \times 10^{-10}$ & $6.6184 \times 10^{-10}$ & $4.7580^{-10}$ & $9.8451^{-11}$ \\
\hline 1.000 & $7.7960 \times 10^{-9}$ & $6.8662 \times 10^{-9}$ & $4.9670^{-9}$ & $1.2351^{-9}$ \\
\hline 10.00 & $1.5901 \times 10^{-7}$ & $1.5922 \times 10^{-7}$ & $1.4395^{-7}$ & $7.5069^{-8}$ \\
\hline \hline
\end{tabular}

the PML layers on the right and top are

$$
\Omega_{R}=\left[X_{r}, X_{r}+D_{r}\right] \times\left[Y_{b}, Y_{t}\right], \quad \Omega_{T}=\left[X_{l}, X_{r}\right] \times\left[Y_{t}, Y_{t}+D_{t}\right],
$$

and the blended corner PML domain is

$$
\Omega_{R T}=\left[X_{r}, X_{r}+D_{r}\right] \times\left[Y_{b}, Y_{t}+D_{t}\right],
$$

where, for $\left(M_{x}, M_{y}\right)=(0.0,0.0)$

$$
X_{l}=-11, \quad X_{r}=2, D_{r}=9, \quad Y_{b}=-11, \quad Y_{t}=2, \text { and } D_{t}=9,
$$

for $\left(M_{x}, M_{y}\right)=(0.4,0.0)$

$$
X_{l}=-7, \quad X_{r}=3, D_{r}=12, \quad Y_{b}=-11, \quad Y_{t}=2, \quad \text { and } D_{t}=9,
$$

for $\left(M_{x}, M_{y}\right)=(0.8,0.0)$

$$
X_{l}=-3, \quad X_{r}=4, D_{r}=15, \quad Y_{b}=-11, \quad Y_{t}=2, \quad \text { and } D_{t}=9,
$$

and for $\left(M_{x}, M_{y}\right)=(0.8,0.4)$

$$
X_{l}=-3, \quad X_{r}=4, D_{r}=15, \quad Y_{b}=-7, \quad Y_{t}=3, \text { and } D_{t}=12 .
$$

In all cases both $\sigma_{R}(x)$ and $\sigma_{T}(y)$ have the same functional form that was used above for a single PML layer on the right. As for Table 1, the reported data is the maximum absolute difference in the numerical domain at $t=10$ between the solution with the PML treatments and a solution on a large domain with BED boundaries, so that the propagated disturbance in the BED numerical domain never reaches the outer boundaries. The pressure solutions with the expanded BED numerical domains for all of these blended corner cases are $O\left[10^{-4}\right]$. In all cases, the results in Table 2 with PML B treatments on the right and the top, and a blended PML B corner treatment are very similar to the results in Table 1 where there is only one PML B on the right. Notice that the data for the $\left(M_{x}, M_{y}\right)=(0.0,0.0)$ case in Table 2 is identical to the comparable data in Table 1 for $M_{x}=0.0$. The disturbances introduced with a blended corner PML treatment for the $\left(M_{x}, M_{y}\right)=(0.4,0.0)$ and $\left(M_{x}, M_{y}\right)=(0.8,0.0)$ cases in Table 2 are of the same order as the disturbances for the comparable $M_{x}=0.0$ cases in Table 1, which would be the expected disturbance levels introduced by the single PML B treatment along the top of the numerical domain with $M_{y}=0.0$. The disturbances introduced with the blended corner PML treatment for the skew $\left(M_{x}, M_{y}\right)=(0.8,0.4)$ case in Table 2 are smaller than the comparable case in Table 1 for either the $M_{x}=0.4$ or the $M_{x}=0.8$ cases. We note that the disturbance introduced into the numerical domain solution by a single PML B layer is actually smaller as the convection speed toward the layer increases, and that the speed in this skew case is approximately 0.89. The data in Table 2 shows that the blended corner PML B treatment introduces essentially no new disturbance into the numerical domain solution beyond what is caused by the one dimensional PML B layers on the right or the top of the numerical domain. 


\section{The Effect of the Outer Boundary}

Reflection from an outer boundary back through a PML into the numerical domain is another distinct problem with its own difficulties. In essence, the outer boundary of a PML layer presents the same problem as the original artificial boundary, only removed a distance from the numerical domain. What is a good transparent boundary condition anyway? The entire PML enterprise has been created because there is no easy answer to this question. The hope in using a PML is that a propagating disturbance will vanish before the outer boundary is reached. The problem in using a PML is that even though there is damping through the layer from the numerical domain towards the outer boundary, there can also be amplification from the outer boundary back towards the numerical domain.

We consider here a range of three simple Boundary Conditions for an outer boundary for a PML. The first is trivial, simply setting all disturbance data to 0 at the outer boundary. We will call this first outer boundary treatment $O B_{1}$. The second outer boundary treatment is to extrapolate $R=p+u$ and $v$ at the outer outflow boundary, and to set $L=p-u=0$. If the outer boundary is at $X_{o b}=X_{r}+D_{r}$, then the data at $X_{o b-1}=X_{o b}-\triangle x$ and $X_{o b-2}=X_{o b}-2 \triangle x$ is interpolated with a cubic Hermite interpolant. This interpolant is used to extrapolate the data at $X_{o b}$, with

$$
f\left(X_{o b}\right)=-4 f\left(X_{o b-1}\right)+5 f\left(X_{o b-2}\right)+\triangle x\left[4 f_{x}\left(X_{o b-1}\right)+2 f_{x}\left(X_{o b-1}\right)\right],
$$

and

$$
f_{x}\left(X_{o b}\right)=12\left[-f\left(X_{o b-1}\right)+f\left(X_{o b-2}\right)\right] /(\triangle x)+8 f_{x}\left(X_{o b-1}\right)+5 f_{x}\left(X_{o b-2}\right) .
$$

Two interpolation problems are solved for each of $f=R$ and $f=v$, one that gives $f$ and $f_{x}$, and another that gives $f_{y}$ and $f_{x y}$. The physical variables $p$ and $u$, and their $x, y$ and $x y$ derivatives, are all recovered at the outer boundary from the extrapolated data for $R$ and the zeroed data for $L$. We will call this second outer boundary treatment $O B_{2}$. The third outer boundary treatment is to propagate $R$ and $v$ with interior differencing, with $L=0$. The propagation equations with damping are

$$
\begin{gathered}
\frac{\partial R}{\partial t}+\left(M_{x}+1\right) \frac{\partial R}{\partial x}+M_{y} \frac{\partial R}{\partial y}+\frac{\partial v}{\partial y}+\sigma_{R}\left(M_{x}+1\right) R=0, \\
L=0 \\
\frac{\partial v}{\partial t}+M_{x} \frac{\partial v}{\partial x}+M_{y} \frac{\partial v}{\partial y}+\frac{1}{2} \frac{\partial R}{\partial y}+\sigma_{v} M_{x} v=0 .
\end{gathered}
$$

Note that at this outer boundary the term $(1 / 2) \partial L / \partial y$ has been dropped from the equation for $v$. We will call this third outer boundary treatment $O B_{3}$. Note that the constraint $L=0$ is stronger than the constraint $L_{x}=0$.

\subsection{PML B on One Side}

In this section we consider simulations with PML B on one side in order to see what can happen to a numerical solution because of the reflection of a signal from the outer boundary of a PML. We consider only the case $\left(M_{x}, M_{y}\right)=(0,0)$ with no convection. Each computation uses PML B, with outer boundary treatments $O B_{i}$, for $i=1,2$, or 3 . All computations have the PML interface at $X_{r}=2$. All of the domain configurations are designed so that the propagating signal does not reach the outer boundaries on the left, top, and bottom.

The data in Table 3 is from three sets of computations, at two different times with two different PML widths. As before, the reported data is the maximum disturbance in the pressure in the numerical domain as calculated by taking the difference between the numerical solution with the PML and a numerical solution with a Big Enough Domain that has no layer, and consequently no interaction with any outer boundary. We note that the first and second sets of data have the same layer width at different times, and the second and third set have different layer widths at the same time. The first set is with PML width $D_{r}=5$ at $t=15$, with $X_{l}=16=Y_{b}=Y_{t}$. The outer boundary of the PML is at $X_{o b}=7$, so that by $t=15$ the signal will propagate to the outer boundary, and any reflection will have a chance to return back through the PML and reach as far as $x=-1$, well within the numerical domain. We note that all of the accuracy 
Table 3: Maximum absolute disturbance from an outer boundary.

PML B with $\triangle x=1 / 24=\triangle y, \triangle t=1 / 48$.

\begin{tabular}{|r|lll|}
\hline $\mathrm{c}$ & $O_{1}$ & $O_{1}$ & $O B_{2}$ \\
\hline \hline & \multicolumn{3}{|c|}{$t=15$ and $w=5$} \\
\hline 0.100 & $9.3051 \times 10^{-6}$ & $8.3402 \times 10^{-6}$ & $9.3046 \times 10^{-6}$ \\
\hline 1.000 & $9.2273 \times 10^{-6}$ & $8.2684 \times 10^{-6}$ & $9.2267 \times 10^{-6}$ \\
\hline 10.00 & $4.7438 \times 10^{-6}$ & $4.2690 \times 10^{-6}$ & $4.7431 \times 10^{-6}$ \\
\hline \hline & \multicolumn{3}{|c|}{$t=25$ and $w=5$} \\
\hline 0.100 & $2.5062 \times 10^{-5}$ & $2.3514 \times 10^{-5}$ & $2.5062 \times 10^{-5}$ \\
\hline 1.000 & $2.4807 \times 10^{-5}$ & $2.3275 \times 10^{-5}$ & $2.4807 \times 10^{-5}$ \\
\hline 10.00 & $1.0290 \times 10^{-5}$ & $9.6544 \times 10^{-6}$ & $1.0289 \times 10^{-5}$ \\
\hline \hline & \multicolumn{3}{|c}{$t=25$ and $w=10$} \\
\hline 0.100 & $4.0624 \times 10^{-6}$ & $3.6134 \times 10^{-6}$ & $4.0623 \times 10^{-6}$ \\
\hline 1.000 & $3.9743 \times 10^{-6}$ & $3.5361 \times 10^{-6}$ & $3.9741 \times 10^{-6}$ \\
\hline 10.00 & $1.7670 \times 10^{-6}$ & $1.7670 \times 10^{-6}$ & $1.7670 \times 10^{-6}$ \\
\hline \hline
\end{tabular}

that was exhibited with this same PML B in Table 1, has disappeared, that all of the disturbances are now $O\left[10^{-6}\right]$. It is worth noting that the data with $c=10$ shows a disturbance that is half what is shown for the smaller damping coefficients. For the cases in Table 1, where the propagating signal never reached an outer boundary, the disturbances introduced by the PML scaled with the damping coefficients, so that the worst performance was with $c=10$, and the effect of introducing a PML could be controlled and made as small as desired by choosing a sufficiently small value for $c$. The second set of data is with PML width $D_{r}=5$ at $t=25$, with $X_{l}=27=Y_{b}=Y_{t}$. The outer boundary of the PML in this case is also at $X_{o b}=7$, so that by $t=25$ the signal will propagate to the outer boundary, and any reflection will have a chance to return back through the PML and reach as far as $x=-11$. The distortions introduced by the PML in this set are approximately twice as large as in the first set above with the same layer width at $t=15$. This is approximately linear growth in time. We note that the $O B_{2}$ extrapolation $\mathrm{BC}$ is slightly better in this case than the other two. The third set is with PML width $D_{r}=10$ at $t=25$, with $X_{l}=27=Y_{b}=Y_{t}$. The outer boundary of the PML in this case is at $X_{o b}=12$, so that by $t=25$ the signal will propagate to the outer boundary, and any reflection will have a chance to return back through the PML and reach as far as $x=-1$. The distortions introduced by the PML in this case are approximately an order of magnitude smaller than in the second case above with a layer that is half as wide at $t=25$. This is an approximately linear decrease with layer width, and shows the advantage of increasing PML width.

Considering all of the data in Table 3, it appears that all of the simple outer boundary treatments that we have tried are equally bad. The disturbances introduced by these simple outer boundary treatments completely obliterate all of the quite good accuracy that was obtained by the PML itself, as demonstrated by the data in Table 1, where an outer boundary was not significant. The disturbances are all in the absolute range of $O\left[10^{-6}\right]$ to $O\left[10^{-5}\right]$, which is approximatrely $1 \%$ to $10 \%$ of the numerical solution with no boundary. In this regard, it is worth noting that an additional series of computations with PML width $D_{r}=5$ at $t=10$ produced disturbances for each value of $c$ that were identical to the data in Table 1, irrespective of the outer boundary treatment, since the reflected signal did not have enough time to propagate back into the numericl domain. We see clearly that the outer boundary can be a significant source of contamination for numerical solutions with artificial boundary treatments. This is at least true for the three outer boundary treatments that we have tried here, which included the extremely crude $O B_{1}$ that simply sets all data to 0 at the outer bounadry, the extrapolation $\mathrm{BC} O B_{2}$, and the $O B_{3}$ that uses interior differenced propagation for the outgoing characteristic variables with the strong Thompson like condition $L=0$ for the incoming characteristic variable. The results from these three outer boundary conditions were very close to each other, typically within about $10 \%$ of each other. The underlying issue undoubtedly is that the PML B both damps going to the right, and amplifies going to the left. However, it remains to be seen if a combination of a more sophisticated outer boundary treatment with a different damping profile can be found that will produce a numerical domain disturbance that can be controlled and made as small as desired. That is another study. 


\subsection{PML B on Four Sides}

In this section we consider simulations with PML B on all four sides, with all four corners. We consider only the case $\left(M_{x}, M_{y}\right)=(0,0)$ with no convection. The complete domain is

$$
\Omega_{C}=\left[X_{l}-D_{l}, X_{r}+D_{r}\right] \times\left[Y_{b}-D_{b}, Y_{t}+D_{t}\right]
$$

with $X_{l}=X_{r}=Y_{b}=Y_{t}=2$, so that the inner numerical domain is the tiny

$$
\Omega_{N}=[-2,2] \times[-2,2],
$$

and the PML widths are all $D_{l}=D_{r}=D_{b}=D_{t}=9$. The complete domain $\Omega_{C}$ is the same as the domain with BED outer boundaries that was used for both the single boundary computations reported in Table 1 , and the blended corner computations reported in Table 2. The outer boundaries are at $|x|=11=|y|$, so that the radiated signal will not reach the outer boundary by $t=10$, and reflections from the outer boundary will return to the PML interfaces by $t=20$.

Table 4: Maximum absolute disturbance with PML on all four sides. PML B with $\triangle x=1 / 24=\triangle y, \triangle t=1 / 48$.

\begin{tabular}{|r|lll|}
\hline $\mathrm{c}$ & $t=10$ & $t=25$ & $t=50$ \\
\hline \hline 0.001 & $1.0653 \times 10^{-12}$ & $9.4618 \times 10^{-7}$ & $3.4832^{-6}$ \\
\hline 0.010 & $1.0829 \times 10^{-11}$ & $9.4618 \times 10^{-7}$ & $3.4829^{-6}$ \\
\hline 0.100 & $1.2589 \times 10^{-10}$ & $9.4601 \times 10^{-7}$ & $3.4806^{-6}$ \\
\hline 1.000 & $3.0775 \times 10^{-9}$ & $9.2995 \times 10^{-7}$ & $3.5239^{-6}$ \\
\hline 10.00 & $2.0769 \times 10^{-7}$ & $2.8321 \times 10^{-7}$ & $3.7187^{-7}$ \\
\hline \hline
\end{tabular}

The experiments reported in Table 4 handle the outer boundary by setting all of the data for all three primitive variables equal to 0 at $|x|=11=|y|$, or with what we have called $O B_{1}$ in the previous section. We note immediately that at $t=10$, before the signal reaches the outer boundary, the disturbance introduced by all four PMLs and corners is of the same order as the error at the same time with just one PML on the right, and even a bit smaller in most cases. At $t=25$ the original signal has propagated through the PML to the outer boundary, and its reflection has returned all the way back across both the PML and the numerical domain. By $t=25$ most of the accuracy of the PML has been lost due to this reflection from the outer boundary, as we have seen in the previous section. Continuing on to $t=50$, the error grows another order of magnitude except for the heavily damped case with $c=10$. With $c=10$ the propagating signal is approximately $O\left[10^{-15}\right]$ at $x-x_{R}=4.75$ in the PML B layer, and approximately $O\left[10^{-35}\right]$ at $x-x_{R}=9.75$, with the signal being damped by approximately five order of magnitude over a PML length of about one and a quarter spatial units. With $c=1$ the propagating signal is approximately $O\left[10^{-5}\right]$ at $x-x_{R}=4.75$ in the PML B layer, and approximately $O\left[10^{-7}\right]$ at $x-x_{R}=9.5$, with the signal being damped by approximately one order of magnitude over a PML length of about two and a half spatial units. For $c=10$, with no flow, a PML B width of $D=9$ appears sufficient to keep the numerical disturbance caused by the PML system from growing in time. For $c=1$, with no flow, a PML width of from $D=27$ to $D=30$ would be required to reduce the propagating signal to $O\left[10^{-15}\right]$. Prohibitvely wide PML B layers would be needed to maintain any accuracy with smaller damping coefficients if such a crude outer boundary is used. We note also that the results reported in Table 4 show for PML B that with sufficient damping for the layer width, the disturbance appears stable in time.

\section{PML C with a Gaussian Jet Flow}

This series of computations is with a parallel Gaussian jet flow for the base solution, with

$$
p_{b}=1, \quad u_{b}=0.8 \exp \left(-36 y^{2}\right), \text { and } ; v_{b}=0 .
$$


Note that the convection velocity is not uniform, though it is a parallel flow, and it does satisfy the Euler equations with uniform density. The maximum convection velocity is +0.8 at the center of the jet. In this case, the perturbation solution of the Linearized Euler Equations satisfies a more general equation form than with a uniform base solution, which produces the constant coefficient LEE. The same pressure source as before is used in the numerical domain but not in the PML domain.

Table 5: Maximum absolute disturbance with a parallel jet. PML C with $\triangle x=1 / 16=\triangle y, \triangle t=1 / 32$.

\begin{tabular}{|r|ll|}
\hline $\mathrm{c}$ & $E_{C}(4)$ & $E_{C}(8)$ \\
\hline \hline 0.001 & $1.16155 \times 10^{-9}$ & $6.74813 \times 10^{-9}$ \\
\hline 0.010 & $1.16102 \times 10^{-8}$ & $6.73600 \times 10^{-8}$ \\
\hline 0.100 & $1.15577 \times 10^{-7}$ & $6.61588 \times 10^{-7}$ \\
\hline 1.000 & $1.10454 \times 10^{-6}$ & $5.60609 \times 10^{-6}$ \\
\hline 10.000 & $7.97674 \times 10^{-6}$ & $3.06075 \times 10^{-5}$ \\
\hline 100.000 & $3.92057 \times 10^{-5}$ & $6.58872 \times 10^{-5}$ \\
\hline \hline
\end{tabular}

The simple PML C damping layer is used for the series of numerical experiments reported in Table 5. For these experiments we have included results with damping coefficient $c=100$. Note that a coarser grid resolution is used for these experiments, with $\triangle x=1 / 16=\triangle y$ and $\triangle t=1 / 32$. The data in the first column is for $t=4$, with numerical domain $\Omega_{N}=[-5,8] \times[-5,5]$, and with PML C layer $\Omega_{R}=[8,14] \times[-5,5]$, with interface $X_{r}=8$. The data in the second column is for $t=8$, with numerical domain $\Omega_{N}=[-9,16] \times[-9,9]$, and with PML C layer $\Omega_{R}=[16,30] \times[-9,9]$, with interface $X_{r}=16$. In both cases, the domains are large enough so that the propagated solution does not reach any outer boundary, either for the numerical domain or for the PML C layer. The data reported in Table 5 is for the maximum absolute disturbance introduced into the numerical solution by the PML C as measured at the interface, where the disturbance is greatest. As before, the data in Table 5 should be scaled by the pressure solution, and along the PML interface there are local wave crests with absolute magnitudes that are $O\left[10^{-4}\right]$. With a parallel jet steady mean flow, the disturbance of the numerical solution that is produced by introducing a PML C on the outflow boundary is comparable to the experiments with a uniform mean flow, ranging in absolute terms from $O\left[10^{-9}\right]$ to $O\left[10^{-5}\right]$, and in relative terms from $O\left[10^{-5}\right]$ to $O\left[10^{-1}\right]$. As in the case of a uniform base flow, the disturbing effect of the PML is controlled by the size of the damping coefficient $c$, and the effect of the PML can be made as small as desired by taking $c$ sufficiently small. For all but the largest two values of $c$, the effect of the PML is invisible at the scale of the solution. We see from these experiments that a PML can be used with nonuniform base flows, and the disturbance that is produced in the numerical solution can be controlled.

\section{Conclusions}

This paper presents the results of a series of numerical experiments to asses and compare PML boundary treatments. Three PML formulations have been used: PML A is an analytic treatment by Hagstrom [13]; PML B is from a characteristic analysis similar to that by Karni [16]; PML C is a familiar damping or sponge zone like that in [20]. PML A is formulated specifically for uniform base flows, and is somewhat more accurate in this case. PML B is simple, and overall is as effective as PML A, while it can be extended to variable coefficient and nonlinear systems. PML C is simple, robust, and the most easily generalized, but the disturbance that it produces in the numerical domain are a couple of orders of magnitude greater than from PML A or B. With a suitable layer width, any of these PML treatments can keep the errors that it introduces to less than $1 \%$ of the solution. All of the reported data is from computations with a uniform flow, except for one series of experiments with a parallel jet base flow. The damping profile $\sigma$ that we have used is smooth at the PML interface, and asymptotes to $\sigma=1$. The results from the reported numerical experiments show that for the Linearized Euler Equations:

1. The level of distortion in the numerical solution that is introduced by each of the PML treatments is essentially linearly dependant upon the scaling coefficient $c$. The distortion levels introduced by the 
PML treatments range from $O\left[10^{-10}\right]$ to $O\left[10^{-1}\right]$ in relative terms, and are controlled by the size of c. In this sense the PML treatments work well. PML A and PML B are similar in performance, and both produce disturbances that are orders of magnitude smaller than those produced by PML C.

2. The blending of two PML treatments in a common corner can be done without introducing any additional distortion into the numerical solution domain. This can be done for a wide variety of PML treatments, including PML B and PML C, but it is not clear how this blending method would work for all PML treatments.

3. The outer boundary of a PML layer can cause significant deterioration of the PML performance. Data was given only for PML B, which both damps to the right and amplifies to the left. Three outer boundary treatments were compared: $O B_{1}$ which sets all data to 0 at the outer boundary; $O B_{2}$ which extrapolates $R=P+u$ and $v$, and sets $L=p-u=0$; and $O B_{3}$ which propagates $R$ and $v$ with interior differencing while setting $L=0$. The errors introduced by the outer boundary conditions decreased linearly with the layer width, but they overwhelmed the PML treatments with the layer thicknesses that we considered except for the strongest damping with $c=10$.

4. The performance of PML C is essentially the same with uniform and nonuniform parallel base flows.

The PML treatments that we have considered result either from operator approximation, which is difficult in the variable coefficient or nonlinear cases, or from eigenvector analysis, which is inherently one dimensional and misses the essentially multidimensional dynamic of systems like the Linearized Euler Equations in two or three space dimensions. All of the results that we have reported are dependant upon the accuracy of the code implementations that have been used, and while validation has been attempted, it is not a proof of coding correctness. Areas that need further investigation include NonReflecting Boundary Conditions for PML outer boundaries, or modifications to PML treatments that can control the error reflected from an outer boundary.

\section{References}

[1] Daniel J. Bodony, "Analysis of sponge zones for computational fluid dynamics," J. Comp. Phys. 212 (2006) pp681-702.

[2] Tim Colonius, "Modeling Artificial Boundary Conditions for Compressible Flow," Annu. Rev. Fluid Mech. 36 (2004) pp315-345.

[3] Tim Colonius, Sanjiva K. Lele, "Computational aerocoustics: progress on nonlinear problems of sound generation," Prog. Aerospace Sciences 40 (2004) pp345-416.

[4] Maria Fröling, A Numerical Investigation of a Perfectly Matched Layer for Computational Aero Acoustics, TRITA-NA-E04041, Master's Thesis, Royal Institute of Technology, Stockholm Sweden (2004).

[5] Dan Givoli, "Non-reflecting Boundary Conditions," J. Comp. Phys. 94 (1991) pp1-29.

[6] Dan Givoli, "High-order local non-reflecting boundary conditions: a review," Wave Motion 39 (2004) pp319-326.

[7] J. W. Goodrich, "An Approach to the Development of Numerical Algorithms for first Order Linear Hyperbolic Systems in Multiple Space Dimensions: The Constant Coefficient Case," NASA TM 106928 (1995), and

[8] J. W. Goodrich, "Accuret Finite Difference Algorithms," NASA TM 107377 (1995).

[9] J. W. Goodrich, "High Accuracy Finite Difference Algorithms for Computational Aeroacoustics," AIAA 97-1584, The 3rd AIAA/CEAS Aeroacoustics Conference, (Atlanta, GA, May, 1997).

[10] J. W. Goodrich, "A comparison of Numerical Methods for Computational Aeroacoustics," AIAA 991943, The 5th AIAA/CEAS Aeroacoustics Conference, (Bellevue, WA, May, 1999). 
[11] J. W. Goodrich, T. Hagstrom, J. Lorenz, "Hermite Methods for Hyperbolic Initial-Boundary Value Problems," Math. Comp. 75 (January, 2006) pp595-630.

[12] Thomas Hagstrom, "Radiation Boundary Conditions for the Numerical Simulation of Waves," Acta Numerica 8 (1999) pp47-106.

[13] Thomas Hagstrom, A New Construction of Perfectly Matched Layers for Hyperbolic Systems with Applications to the Linearized Euler Equations, in "Proceedings of the Sixth International Conference on Mathematical and Numerical Aspects of Wave Propagation," Finland, E. Heikkola, P. Neittaanmki, G. C. Cohen, P. Joly, eds., pp. 125-129, Springer Verlag (2003).

[14] Fang Q. Hu, "Absorbing Boundary Conditions," Int. J. Comp. Fluid Dyn. 18 (2004) pp513-522.

[15] Moshe Israel, Steven A. Orszag, "Approximation of Radiation Boundary Conditions," J. Comp. Phys. 41 (1981) pp115-135.

[16] Smadar Karni, "Far-Field Filtering Operators for Suppression of Reflections from Artificial Boundaries," SIAM J. Numer. Anal. 33 (1996) No.3, pp1014-1047.

[17] Sanjiva K. Lele, "Computational Aeroacoustics - A review," AIAA-97-0018 (1997).

[18] M. Motamed, PML Methods for Aero Acoustics Computations, TRITA-NA-E03108, Master's Thesis, Royal Institute of Technology, Stockholm Sweden (2003).

[19] Peter G. Petropoilos, Li Zhao, and Andreas C. Cangellaris, "A Reflectionless Sponge Layer Absorbing Boundary Condition for the Solution of Maxwell's Equations with High-Order Staggered Finite Difference Schemes," J. Comp. Phys. 139 (1998) pp184-208.

[20] E. Romenski, V. A. Titarev, and E. F. Toro, "Perfectly Matched Layers with High Rate Damping for Hyperbolic Systems," CFD J. 15 (2006) No. 2, pp240-246.

[21] James A. Sochacki, Absorbing Boundary Conditions Using Regions with Exponential Decay, in "Mathematical and Numerical Aspects of Wave Propagation Phenomena," Proceedings of the First International Conference on Mathematical and Numerical Aspects of Wave Propagation, (Strasbourg, France, April 23-26, 1991). Finland, Gary Cohen, Laurence Haloern, Patrick Joly, eds., pp. 293-302, SIAM (1991).

[22] Christopher K. W. Tam, "Computational Aeroacoustics: Issues and Methods," AIAA-97-1774 (1997).

[23] Christopher K. W. Tam, "Advances in Numerical Boundary Conditions for Computational Aeroacoustics," AIAA J. 33 (1995) No. 10, pp1788-1796.

[24] Kevin W. Thompson, "Time Dependent Boundary Conditions for Hyperbolic Systems," J. Comp. Phys. 68 (1987) pp1-24.

[25] Kevin W. Thompson, "Time Dependent Boundary Conditions for Hyperbolic Systems, II" J. Comp. Phys. 89 (1990) pp439-461.

[26] Semyon V. Tsynkov, "Numerical solution of problems on unbounded domains. A review.," Applied Num. Math. 27 (1998) pp465-532.

[27] Meng Wang, Jonathan B. Freund, and Sanjiva K. Lele, "Computational Prediction of Flow-Generated Sound," Annu. Rev. Fluid Mech. 38 (2006) pp483-512. 